\title{
Dimensional characterizations of gender diversity are associated with higher polygenic propensity for cognitive performance in a neurodiverse sample
}

\author{
Taylor R. Thomas ${ }^{1}$, Ashton J. Tener ${ }^{1}$, Ji Seung Yang ${ }^{2}$, John F. Strang ${ }^{3}$, Jacob J. Michaelson ${ }^{1,4,5^{*}}$ \\ 1 Department of Psychiatry, University of Iowa, Iowa City, IA \\ 2 Human Development and Quantitative Methodology, University of Maryland, College Park MD \\ 3 Gender and Autism Program, Center for Neuroscience, Children's National Hospital, George Washington \\ University School of Medicine, Washington DC \\ 4 Iowa Neuroscience Institute, University of Iowa, Iowa City, IA \\ 5 Hawkeye Intellectual and Developmental Disabilities Research Center (Hawk-IDDRC), University of Iowa, \\ Iowa City, IA \\ * jacob-michaelson@uiowa.edu
}

\begin{abstract}
Both sex and gender are characteristics that play a key role in risk and resilience in health and well-being. Current research lacks the ability to quantitatively describe gender and gender diversity, and is limited to endorsement of categorical gender identities, which are contextually and culturally dependent. A more objective, dimensional approach to characterizing gender diversity will enable researchers to advance the health of gender-diverse people by better understanding how genetic factors interact to determine health outcomes. To address this research gap, we leveraged the Gender Self-Report (GSR), a questionnaire that captures multiple dimensions of gender diversity. We then performed polygenic score associations with brain-related traits like cognitive performance, personality, and neuropsychiatric conditions. The GSR was completed by $\mathrm{N}=818$ independent adults with or without autism in the SPARK cohort, and GSR factor analysis identified two factors: Binary (divergence from gender presumed by designated sex to the opposite) and Nonbinary (divergence from male and female gender norms) Gender Diversity (BGD and NGD, respectively). We performed polygenic associations (controlling for age, sex, and autism diagnostic status) in a subset of $\mathrm{N}=452$ individuals and found higher polygenic propensity for cognitive performance was associated with greater BGD $(\beta=0.017, p=0.049)$ and NGD $(\beta=0.036, p=0.002)$, and higher polygenic propensity for educational attainment was also associated with greater $\operatorname{NGD}(\beta=0.030, p=0.015)$. We did not observe any significant associations with personality or neuropsychiatric polygenic scores in this sample. Overall, our results suggest cognitive processes and gender diversity share overlapping genetic factors, indicating the biological utility of the GSR while also underscoring the importance of quantitatively measuring gender diversity in health research contexts.
\end{abstract}

\section{Introduction}

Sex and gender can have major impacts on health outcomes 1] by impinging on the underlying molecular mechanisms of disease and well-being 2]. In health research, designated sex at birth is a more straightforward variable to include than gender, which is diverse in multiple dimensions and is complex to adequately characterize. Gender diversity, which, like designated sex, is a normal manifestation of human diversity, is an especially crucial variable to incorporate in mental health and neuropsychiatric research. Groups that express higher levels of gender diversity than the cisgender proportional majority, such as the LGBTQ+ (lesbian, gay, bisexual, transgender, and queer) community, often have greater rates of anxiety and depression and are more likely to attempt suicide [3]. It has also been shown that discrimination and resilience mediate these negative outcomes in LGBTQ+ college students [4], but the contribution of genetic factors is unknown. Importantly, variation in gender diversity is not uniquely confined to transgender or nonbinary identities. People who identify as cisgender also exhibit variation in gender diversity and expression [5] that would be lost in studies only reporting categorical descriptors of gender. Therefore, a quantitative, dimensional characterization of gender that is independent of categorical gender self-endorsement will enable genetic 
researchers to appropriately incorporate gender diversity in their analyses. Ultimately, this will advance the health of gender diverse people through a greater understanding of how genetic factors interact with gender diversity in determining health outcomes. Because an individual's personal identity informs gender, it follows that gender experience is enabled and shaped by the brain. Therefore, if gender and gender diversity interact with genetic propensity in determining health outcomes, then brain-enabled traits like cognitive performance, personality, and neuropsychiatric conditions may offer insight into how these biological and experiential factors interact to promote well-being.

To address this question, we collected data from $\mathrm{N}=818$ adults regarding their gender identity and expression and sexual orientation. Additionally, the participants filled out the Gender Self-Report, a 30-item self-report questionnaire that captures multiple dimensions of gender diversity. The study participants were recruited from the SPARK cohort 6], which is nationwide genetic study that includes over 270,000 individuals with and without autism. Previous sociological studies have shown there is an enrichment in gender diversity [7] in autistic samples as compared to the general population. Likewise, general population samples of individuals who are transgender or nonbinary are more likely to be autistic and have elevated autistic traits 8. This extensive prior research showing the overlap of autism and gender diversity makes SPARK the ideal cohort for understanding the genetic factors that contribute to gender diversity.

\section{Materials and methods}

\subsection{SPARK cohort description}

SPARK [6] is a United States-based nationwide autism study of over 270,000 individuals. Independent adults in SPARK, with or without autism, were invited to an online Research Match in which they were given the Gender Self Report (GSR) and also asked other questions regarding their sexual orientation, gender identity, and gender expression. This Research Match study was approved by the University of Iowa IRB (IRB\# 201611784) and SPARK is approved by the Western IRB (IRB\# 20151664). All participants provided informed consent.

\subsection{Derivation of Gender Self Report (GSR) factors}

The Gender Self-Report (GSR) itemset was developed through a multi-step multi-input community driven process with autistic cisgender, autistic gender-diverse, and non-autistic cisgender and gender-diverse collaborators. A purposive sampling approach was employed across 8 separate U.S. national recruitments $(\mathrm{N}=1,654)$ to maximize the breadth of the GSR calibration sample and enrich the sample based on the following key characteristics: ASD, gender-diverse identities (binary and nonbinary), the intersection of ASD and gender-diverse identities, transition age/young adult age, and female designation at birth within the entire sample and within ASD, specifically. This purposive sampling resulted in an overall calibration sample that was $37.5 \%$ autistic, $32.6 \%$ gender diverse, and $38.9 \%$ cisgender sexual minority. Two-dimensional normal mixture with graded response model adequately fit the data and yielded two valid and reliable factors: Female-Male Continuum (F-MC) and Nonbinary Gender Diversity (NGD). A transformation of the F-MC scores based on designated sex at birth produced Binary Gender Diversity (BGD) scores (i.e., the distance on the binary gender spectrum from individual's designated sex at birth). GSR calibration utilized differential item functioning, an equity-based psychometric method to identify and reduce bias, in this case by age as well as autism status. Empirical reliability coefficients for response pattern EAP scores were 0.75 for Nonbinary Gender Diversity and 0.85 for Binary Gender Diversity. GSR factors performed well across the following validation metrics: (1) construct validity; GSR scores followed expected score patterns comparing gender identity subgroups, (2) convergent validity; GSR scores correlated with existing gender-related measures and in expected directions, and (3) ecological validity; GSR scores aligned with report of gender-related treatment requests/receipt. These two factors, BGD and NGD, are the two phenotypes used in the the subsequent analyses.

\subsection{Genotype quality control and imputation}

Version 3 Freeze (2019) and Version 4 (2020) genotypes were first merged using PLINK 9]. The merged genotypes were then lifted from hg38 to hg19 using the LiftOver tool 10]. The merged genotypes included 43,209 individuals and 616,321 variants and were then quality controlled using the BIGwas quality control pipeline [11. The default parameters were used, except for skipping Hardy-Weinberg tests and including the flag due to the SPARK cohort being family-based and not a general population sample. The pre-QC annotation step removed 21 variants $(\mathrm{N}=$ 616,299 variants remaining). The SNP QC step removed 101,600 variants due to missingness at a threshold of 0.02 (N $=514,699$ variants remaining). The sample QC step removed 1,114 individuals due to missingness, 67 individuals due 
to heterozygosity, and 176 due to duplicates (monozygotic twins). An additional 9,533 individuals were removed due to genetic ancestry from principal component projections $(\mathrm{N}=32,422$ individuals remaining). The $\mathrm{QC}$ 'd set of $\mathrm{N}=$ 514,699 variants and $\mathrm{N}=32,422$ individuals were then imputed to the TopMed [12 reference panel using the Michigan Imputation Server 13 with the phasing and quality control steps included and to output variants with imputation quality $\mathrm{r} 2>0.3$. After the genotype imputation, the variants were filtered to only the HapMap SNPs (N $=1,054,330$ variants) with imputation quality $\mathrm{r} 2 \geq 0.8$ using bcftools $[14$. Next, they were lifted over from hg38 to hg19 using the VCF-liftover tool (https://github.com/hmgu-itg/VCF-liftover) and the alleles normalized to the hg19 reference genome. Finally, the files were converted to PLINK files with $\mathrm{N}=1,018,200$ final variants.

\subsection{Polygenic score (PGS) calculations}

Polygenic scores were calculated using LDpred2 15 and the bigsnpr tools 16 in $\mathrm{R}$ [17. Because SPARK is family-based, an external LD reference based on 362,320 European individuals of the UK Biobank (provided by the developers of LDpred2) was used to calculate the genetic correlation matrix, estimate heritability, and calculate the infinitesimal beta weights. Polygenic scores were calculated from the following genome-wide association studies performed by the Psychiatric Genomics Consortium: ADHD (2019) [18, autism (2019) 19, bipolar disorder (2021) 20, anorexia nervosa (2019) 21], major depression (2019) 22], schizophrenia (2020) 23], and OCD (2018) 24]. Polygenic scores were calculated from genome-wide association studies performed by the Social Science Genetic Association Consortium for cognitive performance (2018) and educational attainment (2018) 25]. The public LDpred2 beta weights from the Polygenic Index Repository 26. were used to calculate polygenic scores for extraversion, neuroticism, openness, risk tolerance, depressive symptoms, loneliness, and subjective well-being.

\subsection{Polygenic score associations with GSR factors}

Phenotypes were available for $\mathrm{N}=818$, and $\mathrm{N}=460$ also had genetic data that passed quality control. This subset was pruned to remove related individuals using GCTA 27] with a relatedness threshold of 0.05 ( $\mathrm{N}=8$ individuals removed, $\mathrm{N}=452$ remaining). The polygenic scores (PGS) for the 452 individuals were then centered to have a mean of 0 and scaled to have a standard deviation of 1 . Polygenic score main effects were obtained by linear modeling with age in months, designated sex at birth, and autism diagnostic status (autism dx) included as covariates: GSR factor $\sim$ age + sex + autism dx + PGS.

\section{Results}

\subsection{Distribution of the two quantitative gender diversity phenotypes}

The demographic characteristics of the SPARK cohort are shown in Table 1 The cohort was majority autistic and designated female at birth. Approximately one-third of the cohort endorsed a non-cisgender gender identity.

\begin{tabular}{lrr}
\hline variable & entire cohort & genetic cohort \\
\hline total N & 818 & 452 \\
$\%$ male & $22 \%$ & $21 \%$ \\
$\%$ autistic & $60 \%$ & $44 \%$ \\
mean age & 37 & 38 \\
$\%$ gender diverse & $34 \%$ & $27 \%$ \\
\hline
\end{tabular}

Table 1. Cohort demographics.

Age in years.

The factor analyses of the Gender Self Report (GSR) generated two factors: Binary Gender Diversity (BGD) and Nonbinary Gender Diversity (NGD). BGD is the divergence from gender presumed by designated sex to the opposite, and NGD is the divergence from male and female gender norms. The distribution of the factor scores for the $\mathrm{N}=818$ individuals are shown in Figure 1 . The factors range from 0 (no gender diversity) to 1 (high gender diversity), with the mode being for both near 0 (Figure 1A). The overall trends show higher gender diversity in females (Figure 1B) and autistic individuals (Figure 1 C). Individuals who self-identify as transgender trended towards higher BGD, and likewise individuals who self-identify as nonbinary and/or gender neutral trended towards higher NGD (Figure11D. Non-heterosexual sexual orientation identities (lesbian, gay, bisexual, pansexual, queer) also trended towards higher BGD and NGD (Figure 1 1 E). 


\subsection{Polygenic score associations with the Gender Self Report factors}

Polygenic score main effects were obtained by linear modeling $(\mathrm{lm})$ with age in months, designated sex at birth, and autism diagnostic status included as covariates: GSR factor $\sim$ age + sex + autism dx + PGS. Autism diagnostic status was included as a covariate because autism is genetically confounded and in our SPARK cohort and is also phenotypically confounded with gender diversity because the non-autistic participants are the parents of an autistic child (see Discussion for greater clarification on this). The beta coefficients from the PGS score term are shown Figure 2A. Higher polygenic scores for cognitive performance were significantly associated with higher BGD (lm: $\beta=$ 0.017, $p=0.049$ ) and NGD ( $\mathrm{lm}: \beta=0.036, p=0.002)$, meaning that polygenic propensity for greater cognitive performance is predictive of elevated BGD and NGD. Additionally, higher polygenic scores for educational attainment were also associated with higher NGD $(\operatorname{lm}: \beta=0.030, p=0.015)$. No neuropsychiatric polygenic scores were significantly associated with gender diversity, although higher obsessive compulsive disorder (OCD) polygenic scores trended towards higher BGD ( $\mathrm{lm}: \beta=0.013, p=0.116)$, and higher bipolar disorder polygenic scores trended towards lower NGD (lm: $\beta=-0.019, p=0.12)$.

\section{Discussion}

We analyzed two quantitative characterizations of gender diversity, Binary Gender Diversity (BGD) and Nonbinary Gender Diversity (NGD), from factor analysis of the Gender Self-Report (GSR) in a neurodiverse sample of $\mathrm{N}=818$ adults in the SPARK autism cohort. In this sample, we found greater gender diversity in female, autistic, and LGBTQ+ identifying individuals. Due to the structure of SPARK, we were only able to collect data from independent adults with autism or non-autistic parents of children with autism. Therefore, the elevated gender diversity in the autistic subset should be interpreted with the major caveat that the non-autistic parents were older and presumed to adhere to more traditional gender roles. Still, these results are in line with rigorous prior research that has shown the enrichment for gender diversity in autism 7]. Intriguingly, while our results showed higher gender diversity in LGBTQ+ individuals, many people who identify as cisgender also showed evidence of gender diversity. This underscores the value of the GSR in capturing dimensional gender diversity beyond self-endorsed identities. The formation of gender identity is a complex and multi-factorial process [28, and is contextualized by numerous factors like time (e.g., age, generation), region, and culture. Additionally, the conceptualization of these identities requires understanding of how they relate to other points of reference, which can be different for some autistic people 29] who may struggle with understanding social and gender norms.

We performed polygenic score (PGS) associations of GSR factors with brain-related PGS, including cognitive performance, educational attainment, personality traits, and neuropsychiatric conditions in $\mathrm{N}=452$ individuals, $44 \%$ of whom are autistic. Our linear models included age, designated sex at birth, and autism diagnostic status as covariates. For our analyses, we treated autism as a genetic confound with the PGS associations because autism and educational attainment are genetically correlated [19], and the higher autism PGS have been associated with cognitive performance in a general population sample 30. However, future work should analyze the interaction between autism and PGS in their associations with gender diversity. The major limitation of our PGS associations was the small sample size, and hence we were unable to power an analysis stratified on autism diagnostic status. Despite the small sample size, we did observe higher polygenic propensity for cognitive performance was significantly associated with greater gender diversity (BGD and NGD). Higher polygenic propensity for educational attainment was also significantly associated with greater NGD. Overall, these first-of-their-kind results indicate that of the genetic factors that may contribute to gender diversity, the most immediately detectable in our small sample were those related to cognitive performance. This finding may suggest that cognitive capacity is a necessary ingredient in the development of more complex and nuanced gender identities. We expect to see additional associations emerge in future studies with larger sample size that will illuminate both the genetic and environmental/experiential contributions to gender diversity.

\section{Acknowledgments}

We are grateful to all of the individuals and families in SPARK, the SPARK clinical sites, and SPARK staff. We appreciate obtaining access to genetic and phenotypic data for SPARK data on SFARI Base. 
medRxiv preprint doi: https://doi.org/10.1101/2021.11.22.21266696; this version posted November 24, 2021. The copyright holder for this preprint (which was not certified by peer review) is the author/funder, who has granted medRxiv a license to display the preprint in perpetuity. All rights reserved. No reuse allowed without permission.

\section{Data availability statement}

The SPARK genetic data can be obtained at SFARI Base: https://base.sfari.org

The SPARK Research Match data will be available to qualified, approved researchers through SFARI Base upon publication of this article.

\section{Funding}

This work was supported by the National Institutes of Health (MH105527 and DC014489 to JM) and the National Institute of Mental Health (R01MH100028 to JS), as well as grants from the Simons Foundation (SFARI 516716 to JM), the Clinical and Translational Science Award (KL2TR001877 to JM), the Fahs-Beck Fellow Grant to JS, and the National Institutes of Health Predoctoral training grant (T32GM008629 to TT) This work was supported by the University of Iowa Hawkeye Intellectual and Developmental Disabilities Research Center (Hawk-IDDRC) through the Eunice Kennedy Shriver National Institute of Child Health and Human Development (P50HD103556).

\section{Conflict of Interest}

The authors declare that the research was conducted in the absence of any commercial or financial relationships that could be construed as a potential conflict of interest. 
A

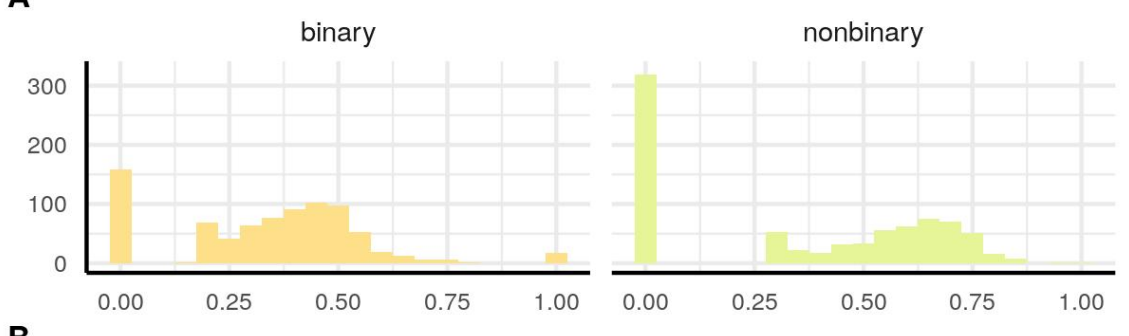

B

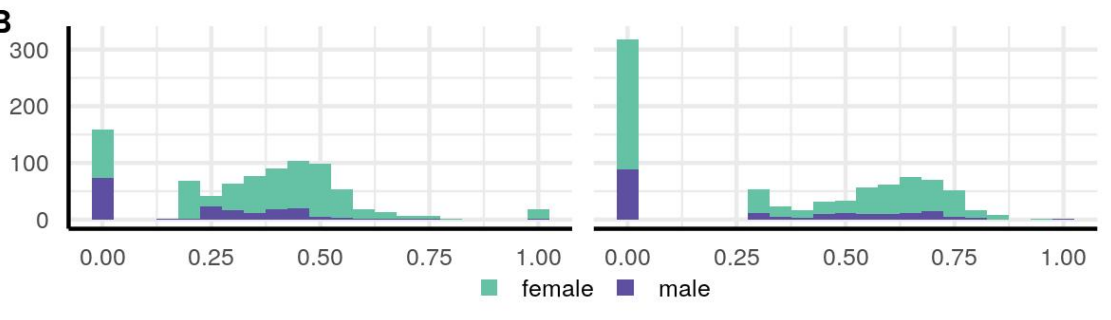

C
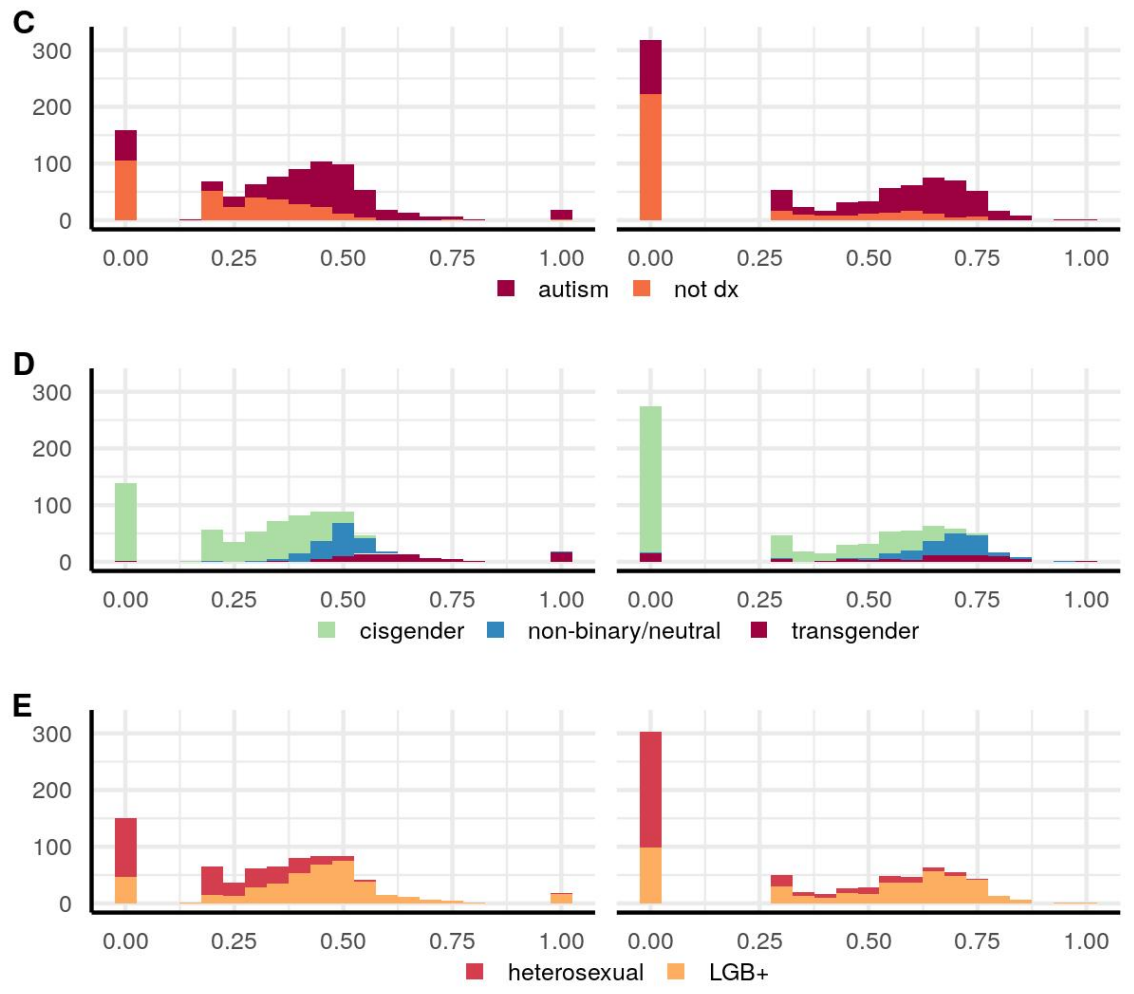

Figure 1. Distribution of the two Gender Self Report (GSR) factors.

A Distribution of the two GSR factors, stratified by $\mathbf{B}$ designated sex at birth, C autism diagnostic status, $\mathbf{D}$ gender identity, and $\mathbf{E}$, sexual orientation. 
medRxiv preprint doi: https://doi.org/10.1101/2021.11.22.21266696; this version posted November 24, 2021. The copyright holder for this preprint (which was not certified by peer review) is the author/funder, who has granted medRxiv a license to display the preprint in perpetuity. All rights reserved. No reuse allowed without permission.

A

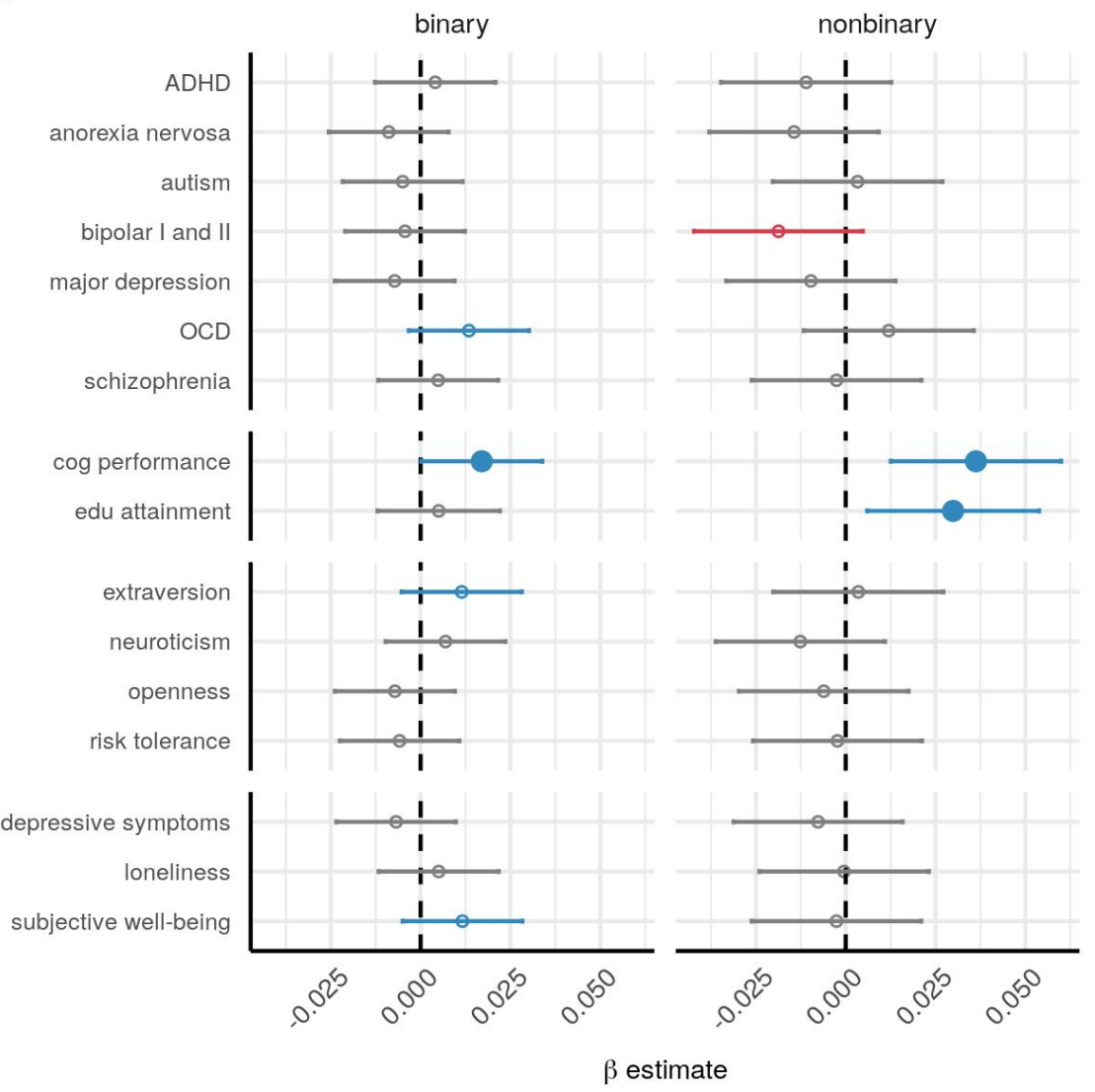

Figure 2. Main effects of polygenic scores (PGS) in linear models predicting the two Gender Self Report (GSR) factors.

A The PGS $\beta$ estimate with the $95 \%$ confidence interval from the linear model GSR factor $\sim$ age + sex + autism $d x+$ PGS is shown. 


\section{References}

1. D. Westergaard, P. Moseley, F. K. H. Sørup, P. Baldi, and S. Brunak, "Population-wide analysis of differences in disease progression patterns in men and women," Nature communications, vol. 10, no. 1, pp. 1-14, 2019.

2. E. A. Khramtsova, L. K. Davis, and B. E. Stranger, "The role of sex in the genomics of human complex traits," Nature reviews. Genetics, vol. 20, pp. 173-190, Mar. 2019.

3. M. P. Marshal, L. J. Dietz, M. S. Friedman, R. Stall, H. A. Smith, J. McGinley, B. C. Thoma, P. J. Murray, A. R. D'Augelli, and D. A. Brent, "Suicidality and depression disparities between sexual minority and heterosexual youth: A meta-analytic review," Journal of adolescent health, vol. 49, no. 2, pp. 115-123, 2011.

4. M. R. Woodford, G. Weber, Z. Nicolazzo, R. Hunt, A. Kulick, T. Coleman, S. Coulombe, and K. A. Renn, "Depression and attempted suicide among lgbtq college students: Fostering resilience to the effects of heterosexism and cisgenderism on campus," Journal of College Student Development, vol. 59, no. 4, pp. 421-438, 2018.

5. T. R. Thomas, D. Hofammann, B. G. McKenna, A. IR van der Miesen, M. A. Stokes, P. Daniolos, and J. J. Michaelson, "Community attitudes on genetic research of gender identity, sexual orientation, and mental health," Plos one, vol. 15, no. 7, p. e0235608, 2020.

6. P. Feliciano, A. M. Daniels, L. G. Snyder, A. Beaumont, A. Camba, A. Esler, A. G. Gulsrud, A. Mason, A. Gutierrez, A. Nicholson, et al., "Spark: a us cohort of 50,000 families to accelerate autism research," Neuron, vol. 97, no. 3, pp. 488-493, 2018.

7. E. Hisle-Gorman, C. A. Landis, A. Susi, N. A. Schvey, G. H. Gorman, C. M. Nylund, and D. A. Klein, "Gender dysphoria in children with autism spectrum disorder," LGBT health, vol. 6, no. 3, pp. 95-100, 2019.

8. V. Warrier, D. M. Greenberg, E. Weir, C. Buckingham, P. Smith, M.-C. Lai, C. Allison, and S. Baron-Cohen, "Elevated rates of autism, other neurodevelopmental and psychiatric diagnoses, and autistic traits in transgender and gender-diverse individuals," Nature communications, vol. 11, no. 1, pp. 1-12, 2020.

9. S. Purcell, B. Neale, K. Todd-Brown, L. Thomas, M. A. Ferreira, D. Bender, J. Maller, P. Sklar, P. I. de Bakker, M. J. Daly, and P. C. Sham, "PLINK: A tool set for whole-genome association and population-based linkage analyses," The American Journal of Human Genetics, vol. 81, pp. 559-575, sep 2007.

10. D. Karolchik, R. Baertsch, M. Diekhans, T. S. Furey, A. Hinrichs, Y. Lu, K. M. Roskin, M. Schwartz, C. W. Sugnet, D. J. Thomas, et al., "The ucsc genome browser database," Nucleic acids research, vol. 31, no. 1, pp. 51-54, 2003.

11. J. C. Kässens, L. Wienbrandt, and D. Ellinghaus, "BIGwas: Single-command quality control and association testing for multi-cohort and biobank-scale GWAS/PheWAS data," GigaScience, vol. 10, June 2021.

12. D. Taliun, D. N. Harris, M. D. Kessler, J. Carlson, Z. A. Szpiech, R. Torres, S. A. G. Taliun, A. Corvelo, S. M. Gogarten, H. M. Kang, et al., "Sequencing of 53,831 diverse genomes from the nhlbi topmed program," Nature, vol. 590, no. 7845, pp. 290-299, 2021.

13. S. Das, L. Forer, S. Schönherr, C. Sidore, A. E. Locke, A. Kwong, S. I. Vrieze, E. Y. Chew, S. Levy, M. McGue, et al., "Next-generation genotype imputation service and methods," Nature genetics, vol. 48, no. 10, pp. 1284-1287, 2016.

14. P. Danecek, J. K. Bonfield, J. Liddle, J. Marshall, V. Ohan, M. O. Pollard, A. Whitwham, T. Keane, S. A. McCarthy, R. M. Davies, et al., "Twelve years of samtools and bcftools," Gigascience, vol. 10, no. 2, p. giab008, 2021.

15. F. Privé, J. Arbel, and B. J. Vilhjálmsson, "Ldpred2: better, faster, stronger," Bioinformatics, vol. 36, no. 22-23, pp. 5424-5431, 2020.

16. F. Privé, H. Aschard, A. Ziyatdinov, and M. G. Blum, "Efficient analysis of large-scale genome-wide data with two r packages: bigstatsr and bigsnpr," Bioinformatics, vol. 34, no. 16, pp. 2781-2787, 2018.

17. R Core Team, R: A Language and Environment for Statistical Computing. R Foundation for Statistical Computing, Vienna, Austria, 2013.

18. D. Demontis, R. K. Walters, J. Martin, M. Mattheisen, T. D. Als, E. Agerbo, G. Baldursson, R. Belliveau, J. Bybjerg-Grauholm, M. Bækvad-Hansen, et al., "Discovery of the first genome-wide significant risk loci for attention deficit/hyperactivity disorder," Nature genetics, vol. 51, no. 1, pp. 63-75, 2019. 
19. J. Grove, S. Ripke, T. D. Als, M. Mattheisen, R. K. Walters, H. Won, J. Pallesen, E. Agerbo, O. A. Andreassen, R. Anney, et al., "Identification of common genetic risk variants for autism spectrum disorder," Nature genetics, vol. 51, no. 3, pp. 431-444, 2019.

20. N. Mullins, A. J. Forstner, K. S. O’Connell, B. Coombes, J. R. Coleman, Z. Qiao, T. D. Als, T. B. Bigdeli, S. Børte, J. Bryois, et al., "Genome-wide association study of more than 40,000 bipolar disorder cases provides new insights into the underlying biology," Nature Genetics, vol. 53, no. 6, pp. 817-829, 2021.

21. H. J. Watson, Z. Yilmaz, L. M. Thornton, C. Hübel, J. R. Coleman, H. A. Gaspar, J. Bryois, A. Hinney, V. M. Leppä, M. Mattheisen, et al., "Genome-wide association study identifies eight risk loci and implicates metabo-psychiatric origins for anorexia nervosa," Nature genetics, vol. 51, no. 8, pp. 1207-1214, 2019.

22. D. M. Howard, M. J. Adams, T.-K. Clarke, J. D. Hafferty, J. Gibson, M. Shirali, J. R. Coleman, S. P. Hagenaars, J. Ward, E. M. Wigmore, et al., "Genome-wide meta-analysis of depression identifies 102 independent variants and highlights the importance of the prefrontal brain regions," Nature neuroscience, vol. 22, no. 3, pp. 343-352, 2019.

23. S. Ripke, J. T. Walters, M. C. O'Donovan, S. W. G. of the Psychiatric Genomics Consortium, et al., "Mapping genomic loci prioritises genes and implicates synaptic biology in schizophrenia," MedRxiv, 2020.

24. P. D. Arnold, K. D. Askland, C. Barlassina, L. Bellodi, O. Bienvenu, D. Black, M. Bloch, H. Brentani, C. L. Burton, B. Camarena, et al., "Revealing the complex genetic architecture of obsessive-compulsive disorder using meta-analysis," Molecular psychiatry, vol. 23, no. 5, pp. 1181-1181, 2018.

25. J. J. Lee, R. Wedow, A. Okbay, E. Kong, O. Maghzian, M. Zacher, T. A. Nguyen-Viet, P. Bowers, J. Sidorenko, R. K. Linnér, et al., "Gene discovery and polygenic prediction from a genome-wide association study of educational attainment in 1.1 million individuals," Nature genetics, vol. 50, no. 8, pp. 1112-1121, 2018.

26. J. Becker, C. A. Burik, G. Goldman, N. Wang, H. Jayashankar, M. Bennett, D. W. Belsky, R. K. Linnér, R. Ahlskog, A. Kleinman, et al., "Resource profile and user guide of the polygenic index repository," Nature human behaviour, pp. 1-15, 2021.

27. J. Yang, S. H. Lee, M. E. Goddard, and P. M. Visscher, "GCTA: A tool for genome-wide complex trait analysis," The American Journal of Human Genetics, vol. 88, pp. 76-82, Jan. 2011.

28. J. D. Bosse and L. Chiodo, "It is complicated: gender and sexual orientation identity in lgbtq youth," Journal of Clinical Nursing, vol. 25, no. 23-24, pp. 3665-3675, 2016.

29. J. F. Strang, A. I. van der Miesen, R. Caplan, C. Hughes, S. daVanport, and M.-C. Lai, "Both sex-and gender-related factors should be considered in autism research and clinical practice," 2020.

30. T.-K. Clarke, M. Lupton, A. M. Fernandez-Pujals, J. Starr, G. Davies, S. Cox, A. Pattie, D. Liewald, L. Hall, D. MacIntyre, et al., "Common polygenic risk for autism spectrum disorder (asd) is associated with cognitive ability in the general population," Molecular psychiatry, vol. 21, no. 3, pp. 419-425, 2016. 